\title{
Laser-Cooling Atoms to Within micro-Kelvins of Absolute Zero
}

\begin{abstract}
Last year's Nobel prize awarded progress in our basic understanding of atom-light interaction. The award was made, on 10 December last year, jointly to Steven Chu, Claude Cohen-Tannoudji, and Bill Phillips for developments of methods to cool and trap atoms with light. Jean Dalibard, who works with Claude Cohen-Tannoudji in the Laboratoire Kastler-Brossel in Paris, takes us through the physics
\end{abstract}

\begin{abstract}
This year's prize rewards a series of outstanding studies that started around 1980 and since have undergone a spectacular expansion - the pioneering work of the three laureates is now used in hundreds of laboratories. Using the interaction of the atoms of a gas with light beams one can control their degrees of freedom and reduce dramatically their thermal motion in order to reach temperatures and confining times which were unthinkable 20 years ago.
\end{abstract}

The process which is at the basis of the manipulation of atoms by light is the recoil when an atom absorbs or emits a photon. For an atom irradiated with resonant light, this recoil velocity is typically between $0.3 \mathrm{~cm} / \mathrm{s}$ and $10 \mathrm{~cm} / \mathrm{s}$. This is very small compared with atomic thermal velocities at room temperature. However, using an intense light source (ie a tunable laser), it leads to considerable forces. Consider, for instance, an atom irradiated with a single resonant laser beam. Each photon absorption increases the atom's velocity in the direction of the beam, and

Like being hit by a tornado, is how Claude Cohen-Tannoudji, right, has described receiving the good news about the Nobel prize. In the lab again, after returning from a week in Stockholm for the prize ceremony, he told $E N$ : $^{\prime}$ When I received the news I was at home preparing one of my course lectures. When I received the call I tried to contact my son and my daughter, they were at lunch; and immediately the journalists came, so I was forced to run into the lab.'

Over the next few weeks,'I tried to maintain my lectures and I think that was good for me because it gave me a fixed reference to follow.

He could not wait to get back to his students and his research; although, this would not be the research of an average physicist. His lectures were on Bose-Einstein condensation, which perhaps we would all like to have attended. And as for his research, micro-Kelvins are not cold enough:'We are trying to develop new methods each spontaneous emission process leads to a recoil in a random direction. The rate for these absorption-spontaneous emission cycles is only limited by the lifetime of the excited atomic level, and it can reach $10^{8}$ per second. After averaging over the direction of the spontaneously emitted photon, the resulting acceleration of the atom is in the direction of the laser beam and it can reach $10^{5}$ times the gravitational acceleration on Earth.

The first spectacular use of this radiation pressure force was achieved by Phillips, Metcalf and their colleagues, at NBS Washington, between 1982 and 1985. They stopped a sodium atom beam, initially travelling at $1000 \mathrm{~m} / \mathrm{s}$, within a distance of 1 meter. A major difficulty in the experiment was circumventing the Doppler shift: as an atom slows down the apparent laser frequency changes and the atom shifts out of resonance. Phillips developed what is now called a Zeeman slower, which is a solenoid with a spatially variable magnetic field. The atomic Bohr

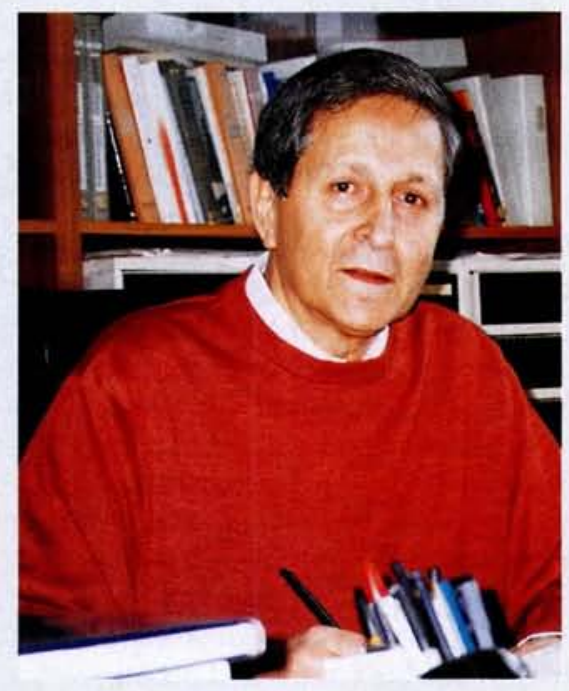

for measuring the temperature. And also, cooling below the single photon recoil limit.' And:'We are frequency now depends on position and the atom-laser resonance can be maintained over the whole decelerating trajectory. At the same time, another group (W. Ertmer, R. Blatt, J. Hall and M. Zhu) also succeeded in stopping an atomic beam by chirping the laser frequency during the deceleration to compensate for the variation due to Doppler shift. By 1985 Phillips could store the stopped atoms in a magnetic trap, of a kind proposed two years earlier by D. Pritchard.

In parallel during this period (19801987), the theoretical description of the manipulation of atoms by light was established. Early work on this topic was performed by the groups of A. Ashkin, D. Cook, and D. Wineland in the USA, and A.P. Kazantsev, V. Lethokov, and S. Stenholm in Europe. Subsequently, Cohen-Tannoudji and his colleagues interpreted the forces acting on an atom in a laser field in terms of 'dressed atomic levels', whose positions vary in space if the light intensity is not uniform. The description of spontaneous emission processes for a dressed atom allowed for a quantitative treatment of the confinement and of the heating in a laser intensity gradient. In particular this led Cohen-Tannoudji and co-workers to the observation of the channelling of atoms near the nodes of a laser standing wave, perpendicular to the atomic beam.

In 1985 Chu and his colleagues in Bell Labs realized the first 'optical molasses'. They used 6 laser beams opposed in pairs and aligned along three orthogonal directions in space. At the intersection of the 6 laser beams they could catch $10^{5}$ sodium

building atomic clocks.'

How was his week in Stockholm? 'Oh, very moving. What I found wonderful was the combination of solemnity and an extremely warm atmosphere. It's a very delicate combination; in my opinion it's unique. The Swedish: we were dressed in formal dress but at the same time they were very warm and very kind.' Editor Other applications of the physics: Optical lattices, in which ultra-cold atoms are arranged in periodic structures created by laser standing waves, have been built. A new lithography technique is being developed in which atoms are deposited onto a substrate with a pattern imposed by guiding laser beams. Last but not least, the cold atoms, with their large de Broglie wavelength, constitute a remarkable source for matter-wave interferometry and for the study of pure quantum statistical phenomena, such as Bose-Einstein condensation.JD 


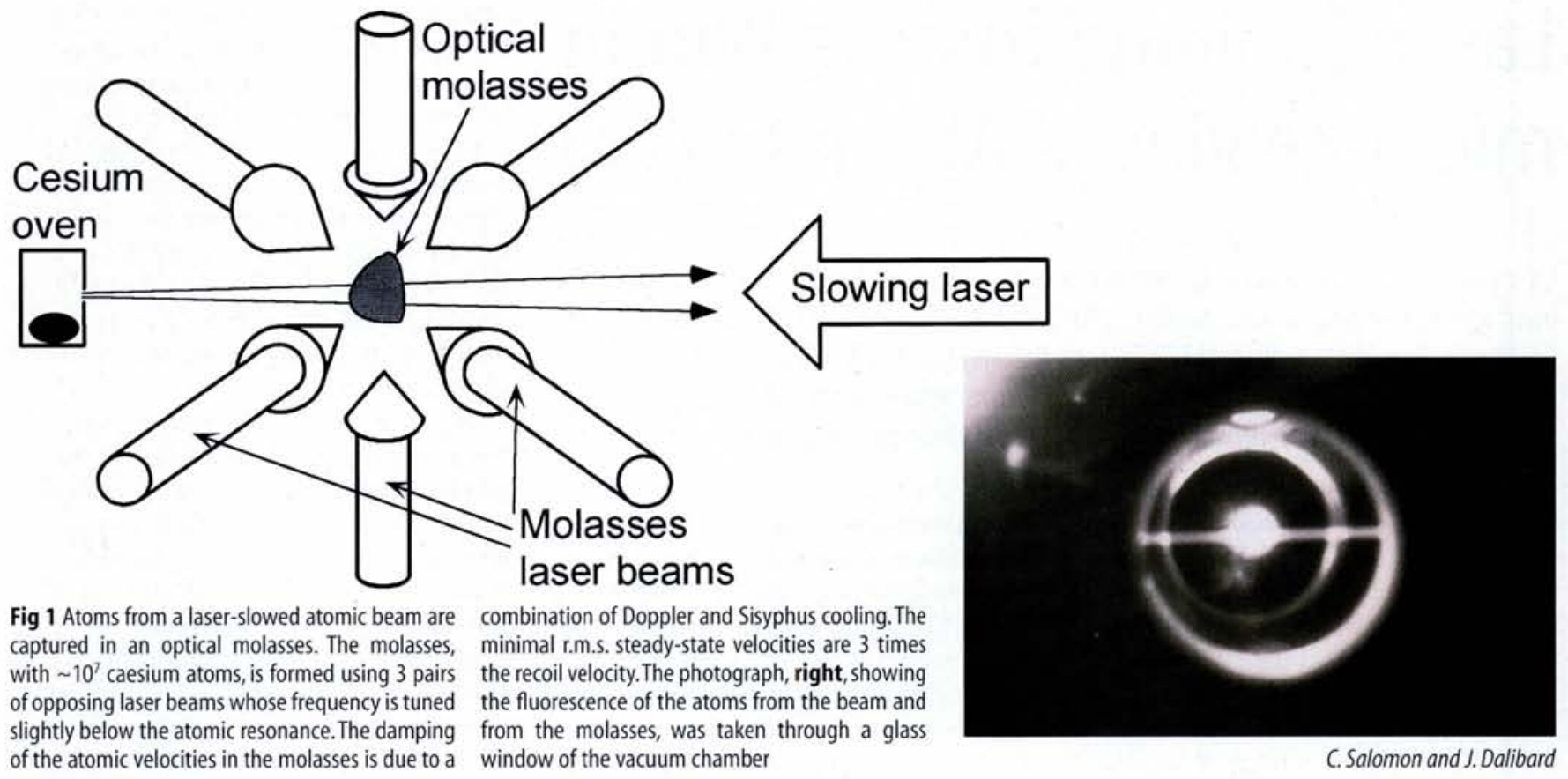

atoms which originated from an atomic beam slowed by radiation pressure. This experiment followed a remarkably elegant suggestion in 1975 by T. Hänsch and A. Schawlow (Stanford) for damping the velocities of an atomic gas using the Doppler effect. In the molasses configuration (figure 1), if the laser frequency is tuned slightly below the atomic resonance frequency, a moving atom always feels a net radiation pressure force opposed to its velocity. Indeed, because of the Doppler effect, a counterpropagating wave is closer to resonance than a copropagating one, and it exerts on the atom a larger radiation pressure force. The atoms then move as in a viscous medium, hence the name optical molasses. The equilibrium velocity distribution is Gaussian, with a rootmean-square velocity actually much larger than the recoil velocity. It corresponds to a balance between the viscous damping described above and the heating due to random recoils in spontaneous emission processes.

The results obtained by $\mathrm{Chu}$ and his colleagues in 1985 confirmed the Doppler cooling theory. Subsequently, Phillips designed a similar experiment and he performed a systematic study of the temperature of the sodium atoms in the molasses. He developed precise methods to analyse the atomic velocity distribution, in particular a time-of-flight analysis using a laser beam located below the optical molasses. He then found, in the spring of 1988, a spectacular violation of Murphy's law: the temperature in the molasses can be much smaller (by a factor 6 for $\mathrm{Na}$ ) than the lowest theoretical limit for Doppler cooling.

The explanation came a few months later from Cohen-Tannoudji and co-workers, and from Chu and his group: a 'real atom', such as sodium, should not be modelled as a simple two-level system. In particular, the atomic ground level consists of several substates, and this leads to a velocity damping much stronger than in Doppler cooling. Because of the polarization gradients which are present in the light field of the optical molasses, the light-shifts (or AC Stark-shifts) of these substates vary in space and they differ from one sublevel to the other. In addition, optical pumping tends to accumulate the atoms into the lowest Zeeman substate. Therefore, as an atom moves in the light field it has to climb a steep potential hill, before an optical pumping process puts it back into another potential valley. The atom is in a situation analogous to Sisyphus who, in the Greek mythology, had been sentenced for life to continuously push a rock to the top of a mountain. The steady-state of Sisyphus cooling is obtained when the atoms are trapped in the potential valleys, with a r.m.s. velocity which can be as low as 3 recoil velocities.

At first sight the recoil limit seems an unavoidably low limit to laser cooling. If the atoms being cooled constantly absorb and emit photons, the randomness of the recoil due to the last emitted photon leads to a dispersion of the atomic velocity at least of order of the recoil velocity, $v_{\mathrm{r}}$ (where $v_{\mathrm{r}}=h / m \lambda, m$ is the atomic mass and $\lambda$ the photon wavelength). However, this limit can be circumvented if the atoms that are almost at rest (ie with a velocity lower than $v_{\mathrm{r}}$ ) are exempted from the absorption-spontaneous emission cycles. Such a situation occurs when an atom with two ground-states, $g_{1}$ and $g_{2}$, and an excited state, $e$, interacts with two coherent laser waves exciting respectively the transitions $g_{1} \leftrightarrow \mathrm{e}$ and $g_{2} \leftrightarrow \mathrm{e}$. In this case there exists a linear combination of $g_{1}$ and $g_{2}$ which is not coupled to the light. This is a 'dark state', discovered in the 1970 s by A. Gozzini and co-workers in Pisa. If the two laser waves do not have the same directions in space, the Doppler effect makes this dark state velocity dependent. It is only for an adjustable velocity class, for instance $v=0$, that the linear combination of $g_{1}$ and $g_{2}$ is truly decoupled from the light.

This sub-recoil cooling was developed by C. Cohen-Tannoudji, A. Aspect, E. Arimondo, and their colleagues in Paris in 1988 , in a one-dimensional geometry. The initial gain with respect to the recoil limit was modest (a factor $\sim 2$ ), but since then the method has been improved in a spectacular way, and r.m.s. velocities as low as $\mathrm{v}_{\mathrm{r}} / 30$ have been measured recently. It has also been extended with success to two and three dimensions. Another sub-recoil method was developed by Chu and coworkers in 1992. It is also based on a zero probability for the laser-excitation of atoms at rest, which is achieved in this case using velocity-selective Raman transitions between two ground atomic levels. 